\title{
Rapid deconjugation of $\mathrm{SN}-38$ glucuronide and adsorption of released free $\mathrm{SN}-38$ by intestinal microorganisms in rat
}

\author{
AKIRA TAKAKURA $^{1}$, AKINOBU KURITA $^{2}$, TAKASHI ASAHARA ${ }^{2}$, MASANORI YOKOBA $^{3}$, \\ MICHIKO YAMAMOTO $^{1}$, SHINICHIRO RYUGE ${ }^{1}$, SATOSHI IGAWA ${ }^{1}$, YUKITOSHI YASUZAWA ${ }^{2}$, \\ JIICHIRO SASAKI $^{1}$, HIROSUKE KOBAYASHI ${ }^{3}$ and NORIYUKI MASUDA ${ }^{1}$ \\ ${ }^{1}$ Department of Respiratory Medicine, Kitasato University School of Medicine, Sagamihara, Kanagawa 252-0374; \\ ${ }^{2}$ Yakult Central Institute for Microbial Research, Kunitachi, Tokyo 186-8650; ${ }^{3}$ Department of Respiratory Medicine, \\ Kitasato University School of Allied Health Sciences, Sagamihara, Kanagawa 252-0373, Japan
}

Received April 15, 2011; Accepted November 24, 2011

DOI: $10.3892 / \mathrm{ol} .2011 .519$

\begin{abstract}
One of the dose-limiting toxicities of irinotecan hydrochloride (CPT-11) is delayed-onset diarrhea. CPT-11 is converted to its active metabolite, $\mathrm{SN}-38$, which is conjugated to SN-38 glucuronide (SN-38G). SN-38G excreted in the intestinal lumen is extensively deconjugated by bacterial $\beta$-glucuronidase, resulting in the regeneration of $\mathrm{SN}-38$, which causes diarrhea. However, the deconjugation of SN-38G by the intestinal microflora remains to be clarified. This study aimed to investigate the microbial transformation of SN-38G by an anaerobic mixed culture of rat cecal microorganisms. Concentrations of SN-38G and SN-38 were then determined using high-performance liquid chromatography. Complete deconjugation of SN-38G to SN-38 in the mixed cultures was observed within $1 \mathrm{~h}$ of incubation, with $62.7 \%$ of the added SN-38G being found in the supernatant. Approximately $80.4 \%$ of the $\mathrm{SN}-38$ in the supernatant was bound to protein, and the remaining $19.6 \%$ was detected as active free SN-38. In total, only $12.3 \%$ (19.6 x $62.7 \%)$ of the SN-38G added to the test tube was found in the supernatant in the ultrafiltrable free form, indicating that approximately $90 \%$ of the SN-38G added to the growth medium either remained adsorbed onto the pelleted fraction or occurred in a protein-bound form in the supernatant. The remaining $10 \%$ of the SN-38G added to the growth medium existed in the unbound form, the form capable of causing damage to the intestinal membrane. In conclusion, these results indicated that the greater part of the $\mathrm{SN}-38$ produced from SN-38G by the action of bacterial $\beta$-glucuronidase is rapidly adsorbed onto intestinal bacterial cell walls or dietary fibers in pelleted fraction, and only $10 \%$ remains in the ultrafiltrable unbound form in the intestinal luminal fluid.
\end{abstract}

Correspondence to: Dr Noriyuki Masuda, Department of Respiratory Medicine, Kitasato University School of Medicine, 1-15-1 Minami-Ku Kitasato, Sagamihara, Kanagawa 252-0374, Japan

E-mail: masuda@med.kitasato-u.ac.jp

Key words: CPT-11, SN-38 glucuronide, intestinal microorganisms, deconjugation, adsorption, diarrhea

\section{Introduction}

Irinotecan hydrochloride (CPT-11) is a water-soluble camptothecin derivative that inhibits topoisomerase I by stabilizing the enzyme-DNA 'cleavable complex', causing double-stranded DNA breaks during DNA replication and, ultimately, cell death (1). CPT-11, administered as a single agent or in combination with other drugs, exhibited significant antitumor activity against various human malignancies, including colorectal, small and non-small cell lung, gastric, cervical and ovarian cancers (2). CPT-11 occasionally causes unpredictable and severe neutropenia and/or diarrhea (3). Late-onset diarrhea remains one of the as-yet unresolved problems linked to irinotecan administration and has the potential to cause life-threatening dehydration and electrolyte imbalance, which may necessitate premature discontinuation of chemotherapy $(2,4)$. The current standard strategy for the treatment of CPT-11-induced delayed-onset diarrhea is administration of high-dose loperamide (5). Other therapeutic measures that have been attempted include i) a Japanese herbal remedy (Kampo medicine) (6) or antibiotics (7-9) that inhibit intestinal $\beta$-glucuronidase (7-9); ii) prevention of reabsorption of SN-38 and CPT-11 by oral alkalization (10) or administration of adsorbents, activated charcoal (11) or AST-120 (12); and iii) various other treatments, including encephalinase inhibitor (13), glutamine (14) and budesonide (15). However, the success of these approaches has been limited.

The hydrophobic active metabolite, SN-38, is generated from CPT-11 by carboxylesterase and then conjugated to yield $\mathrm{SN}-38$ glucuronide $(\mathrm{SN}-38 \mathrm{G})$ by hepatic uridine diphosphate glucuronosyl transferases (UGTs). SN-38G is an inactive metabolite and is secreted in the duodenum (16-18). Deconjugated SN-38 exerts a toxic effect when it comes into contact with the intestinal mucosa, and deconjugation of SN-38G to SN-38 by $\beta$-glucuronidase in the intestinal microflora is considered to be a major contributory factor in the development of CPT-11-induced late-onset diarrhea (11,19-24).

To the best of our knowledge, however, as yet no studies have been conducted on the biotransformation of SN-38G by the intestinal microflora, even though this knowledge is essential in achieving a precise understanding of the mechanism 
by which CPT-11 induces late-onset diarrhea. The purpose of this study was to investigate the microbial transformation of SN-38G by using an anaerobic mixed culture of rat cecal microorganisms.

\section{Materials and methods}

Chemicals. SN-38G [molecular weight (MW): 568.53; Lot no. MS01077] and SN-38 (MW: 410.42; Lot no. 300917R) were kindly donated by Yakult Honsha Co. Ltd. (Tokyo, Japan). Camptothecin was purchased from Sigma (St. Louis, MO, USA). Other chemicals used were of the highest commercially available grade.

Animals. The study was approved by the Institutional Animal Care and Use Committee of Yakult Central Institute. The animals comprised 2 10-week-old male Wistar rats, weighing $271.6 \mathrm{~g}$ and $273.0 \mathrm{~g}$. The animals were obtained from Japan SLC (Hamamatsu, Japan) and reared in cages with a wire-mesh bottom in a temperature- and humidity-controlled animal facility under a 12-h light-dark cycle. The animals received a steam-sterilized commercial diet, and had free access to food and water during the acclimatization period.

Cecal contents. The rats were sacrificed by exsanguination under pentobarbital anesthesia. The cecum was removed and the contents collected and transferred within $5 \mathrm{~min}$ to an anaerobic porter (Terumo, Tokyo, Japan). Cecal contents (4 g) from the two rats were diluted approximately 10-fold in $40 \mathrm{ml}$ sterile, pre-reduced peptone-yeast extract (PY) broth (see below) under flushing with $\mathrm{N}_{2}$.

Culture experiment. The basal medium used in this experiment was PY broth, which consisted of $1 \mathrm{~g}$ polypeptone (Daigo-Eiyo Chemical Co., Tokyo, Japan); 1 g yeast extract (Difco Laboratories, Detroit, MI, USA); $4 \mathrm{ml}$ salt solution recommended by Holdeman et al (25); and $0.05 \mathrm{~g}$ l-cysteine$\mathrm{HCl}$ per $100 \mathrm{ml}$ in $0.02 \mathrm{M}$ phosphate buffer, $\mathrm{pH} 7.5$, sterilized at $120^{\circ} \mathrm{C}$ for $15 \mathrm{~min}$. A 1:10 suspension of the cecal contents in the peptone-yeast extract broth was incubated for a specified period of time under an atmosphere of pure $\mathrm{N}_{2}$ after the addition of SN-38G. The culture medium was then analyzed. Assays were run in triplicate for samples incubated with SN-38G and the data expressed as the mean \pm standard deviation.

SN-38G-containing medium. SN-38G was dissolved in methanol at $1 \mathrm{mg} / \mathrm{ml}$. After sterilization by passage through a $0.45-\mu \mathrm{m}$ membrane filter, $0.3 \mathrm{ml} \mathrm{SN}-38 \mathrm{G}$ solution was added to $30 \mathrm{ml}$ cecal content suspension (1:10). The medium was then dispensed in $1.5-\mathrm{ml}$ aliquots into $15 \times 105-\mathrm{mm}$ test tubes.

Incubation. For anaerobic growth, the tubes containing SN-38G were inoculated under flushing with $\mathrm{N}_{2}$, tightly stoppered and incubated for $0,1,3,6,24,48$, or $72 \mathrm{~h}$ at $37^{\circ} \mathrm{C}$. The time-point immediately following inoculation was denoted as $0 \mathrm{~h}$. The $\mathrm{N}_{2}$ gas was used after removal of any residual oxygen by passing it over heated copper gauze.

Extraction and high-performance liquid chromatographic analysis of $S N-38 G$ and $S N-38$. The inoculated tubes were assayed for $\mathrm{SN}-38 \mathrm{G}$ or its metabolite, SN-38, at various time-points during incubation under $\mathrm{N}_{2}$ for up to $72 \mathrm{~h}$. Spent culture medium $(1 \mathrm{ml})$ was centrifuged at 15,000 rpm for $1 \mathrm{~min}$ at $0^{\circ} \mathrm{C}$ and the supernatant was collected. For the assay of the whole culture medium or its supernatant, $0.1 \mathrm{ml}$ spent culture medium or the supernatant described above was mixed with $0.4 \mathrm{ml}$ methanol and centrifuged at 15,000 rpm for $1 \mathrm{~min}$ at $-10^{\circ} \mathrm{C}$. Each supernatant $(50 \mu \mathrm{l})$ was diluted with $0.2 \mathrm{ml}$ of $0.15 \mathrm{M} \mathrm{H}_{3} \mathrm{PO}_{4}$ and $0.25 \mathrm{ml}$ internal standard solution containing $1 \mu \mathrm{g} / \mathrm{ml}$ camptothecin. To separate non-protein-bound compounds from protein-bound compounds, supernatant ultrafiltrates were obtained by centrifugation at 2,000 $\mathrm{g}$ and $4^{\circ} \mathrm{C}$ for $20 \mathrm{~min}$ in the ultra-free ${ }^{\circledR}-\mathrm{MC} 30,000 \mathrm{NMWL}$ filter unit (Centriplus Millipore Corporation, Bedford, MA, USA), and were then processed by the same means as described for the spent culture medium and supernatant. Each sample was fresh-frozen at $-20^{\circ} \mathrm{C}$ until analysis.

Concentrations of SN-38G and SN-38 were determined using a high-performance liquid chromatographic (HPLC) method with a fully automated on-line solid phase extraction system (Spark Holland, Emmen, The Netherlands) as previously described (26). The quantification limit for SN-38G and SN-38 was $100 \mathrm{ng} / \mathrm{ml}$ and $10 \mathrm{ng} / \mathrm{ml}$, respectively.

For analysis of the concentrations of the lactone and carboxylate forms of $\mathrm{SN}-38,0.3 \mathrm{ml}$ culture medium or its supernatant was mixed for $5 \mathrm{sec}$ with $0.3 \mathrm{ml}$ methanol, which was previously chilled in a dry, ice-cold isopropanol bath and immediately centrifuged at $15,000 \mathrm{rpm}$ for $1 \mathrm{~min}$ at $-10^{\circ} \mathrm{C}$. The obtained supernatant was rapidly poured into a vial, set on an autosampler at $4^{\circ} \mathrm{C}$ and analyzed by the HPLC system. The concentrations of the SN-38 lactone and carboxylate forms were determined using HPLC according to the method reported by Kaneda et al (27). The lower limits of quantification for the SN-38 lactone and carboxylate forms were $1 \mathrm{ng} / \mathrm{ml}$ and $10 \mathrm{ng} / \mathrm{ml}$, respectively.

The percentages of the protein-bound compounds (SN-38 and SN-38G) were determined as follows: i) concentration of protein-unbound compounds in supernatant $=\mathrm{A} \times \mathrm{B} / \mathrm{C}$, where $\mathrm{A}$ is the concentration of the compound in ultrafiltrate, $\mathrm{B}$ is the weight of the ultrafiltrate after centrifugation and $\mathrm{C}$ is the weight of the supernatant applied to the ultrafilter unit; ii) percentage of protein-bound compounds $(\%)=(\mathrm{A}-\mathrm{B}) / \mathrm{A} \times 100$, where $\mathrm{A}$ is the concentration of the compounds in supernatant and $\mathrm{B}$ is the concentration of the protein-unbound compounds in supernatant.

\section{Results}

Deconjugation of SN-38G in the culture of cecal content. Cecal content (diluted 10-fold) was grown anaerobically in PY broth in the presence of SN-38G. Immediately after the start of incubation, the percentage of $\mathrm{SN}-38 \mathrm{G}$ in the supernatant was found to be $46.9 \%$, while in the pelleted fraction it was $13.8 \%$ (Fig. 1). The remaining $39.4 \%$ was identified as deconjugated SN-38 (33.3\% in the supernatant and 6.1\% in the pelleted fraction), reflecting the rapid deconjugating activity of the cecal microflora. At $1 \mathrm{~h}$ after incubation, most of the SN-38G was converted to SN-38 by bacterial $\beta$-glucuronidase, and little SN-38G was subsequently detectable in the culture medium. 
Table I. Percentages of protein-bound SN-38G and SN-38 in the cell-free supernatant ${ }^{\mathrm{a}}$.

\begin{tabular}{|c|c|c|c|c|c|}
\hline & \multirow{2}{*}{$\begin{array}{l}\text { Incubation } \\
\text { time }(\mathrm{h})\end{array}$} & \multicolumn{2}{|c|}{ Composition (\%) } & \multirow{2}{*}{$\begin{array}{l}\text { Protein-bound compound } \\
\text { in supernatant }\end{array}$} & \multirow{2}{*}{$\begin{array}{l}\text { Rate of protein-unbound } \\
\text { compound in supernatant to total } \\
\text { SN-38G added }(\%)^{\mathrm{b}}\end{array}$} \\
\hline & & Sediment & Supernatant & & \\
\hline $\mathrm{SN}-38 \mathrm{G}^{\mathrm{c}}$ & 0 & $13.8 \pm 0.7$ & $46.9 \pm 3.9$ & $81.3 \pm 0.7$ & $8.8 \pm 1.0$ \\
\hline \multirow[t]{7}{*}{ SN-38 } & 0 & $6.1 \pm 1.3$ & $33.3 \pm 2.1$ & $54.1 \pm 1.4$ & $15.3 \pm 0.5$ \\
\hline & 1 & $37.3 \pm 0.2$ & $62.7 \pm 0.2$ & $80.4 \pm 4.4$ & $12.3 \pm 2.7$ \\
\hline & 3 & $42.3 \pm 1.8$ & $57.7 \pm 1.8$ & $86.2 \pm 0.7$ & $8.0 \pm 0.6$ \\
\hline & 6 & $38.9 \pm 1.5$ & $61.1 \pm 1.5$ & $88.2 \pm 1.1$ & $7.2 \pm 0.7$ \\
\hline & 24 & $47.3 \pm 0.7$ & $52.7 \pm 0.7$ & $88.4 \pm 2.0$ & $6.1 \pm 1.1$ \\
\hline & 48 & $52.4 \pm 4.3$ & $47.6 \pm 4.3$ & $91.7 \pm 0.4$ & $3.9 \pm 0.2$ \\
\hline & 72 & $55.4 \pm 2.6$ & $44.6 \pm 2.6$ & $85.8 \pm 1.0$ & $6.4 \pm 0.7$ \\
\hline
\end{tabular}

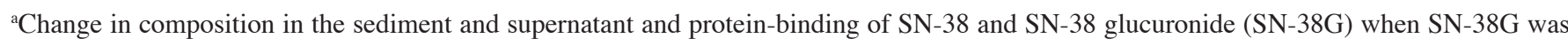
added at $10 \mu \mathrm{g} / \mathrm{ml}$ to mixed cecal content culture carried out under anaerobic conditions. ${ }^{b}$ Rate of protein-unbound compound in supernatant to total $\mathrm{SN}-38 \mathrm{G}$ added $=($ composition in supernatant $) \mathrm{x}(1-$ protein-bound compound in supernatant $/ 100) .{ }^{\mathrm{c}}$ At $1 \mathrm{~h}$ after incubation, the majority of SN-38G was converted to SN-38 by bacterial $\beta$-glucuronidase, and little SN-38G was subsequently detected in the culture medium. Data are expressed as the mean $\pm \mathrm{SD}$ of the experiment performed in triplicate.

Change in percentage concentration of lactone and carboxylate forms of $\mathrm{SN}-38$ during the study period. Immediately following the addition of SN-38G to the culture medium, the metabolites (deconjugated SN-38) were in the lactone form, irrespective of whether they were derived from the pelleted fraction or the supernatant (Fig. 1). At $1 \mathrm{~h}$ after the start of incubation, 54.4 and $37.0 \%$ of the total SN-38 was found in the lactone form, and 8.3 and $0.3 \%$ in the carboxylate form in the pelleted fraction and supernatant, respectively. From 3 up to $72 \mathrm{~h}$ of incubation, the lactone to carboxylate ratio remained relatively constant at 9:1. In the supernatant, the lactone-to-carboxylate ratio was $85: 15$, with this ratio remaining stable up to $72 \mathrm{~h}$ (Fig. 1).

Change in the percentage composition of $\mathrm{SN}-38$ in the pelleted fraction and supernatant during the study period. Immediately following the addition of SN-38G, 19.9\% was found in the pelleted fraction $(13.8 \%$ as $\mathrm{SN}-38 \mathrm{G}$ and $6.1 \%$ as SN-38 lactone) (Fig. 1). After 1 h of incubation, 37.0 and $0.3 \%$ in the pelleted fraction, and 54.4 and $8.3 \%$ in the supernatant was found in the lactone and carboxylate forms of $\mathrm{SN}-38$, respectively. The percentage of the lactone form of SN-38 in the pelleted fraction gradually increased from $40.8 \%$ after $3 \mathrm{~h}$ to $52.8 \%$ after $72 \mathrm{~h}$ of incubation. The bulk of the SN-38 in the pelleted fraction was in the lactone form, which may be the main form adsorbed onto bacterial cells in the pelleted fraction due to its lipophilic nature. Almost no metabolites were detected in the carboxylate form in the pelleted fraction.

Binding of $S N-38 G$ and $S N-38$ to protein in cell-free supernatant. The percentages of protein-bound SN-38G and SN-38 in the cell-free supernatant are shown in Table I. Immediately after the start of incubation, $81.3 \%$ of $\mathrm{SN}-38 \mathrm{G}$ and $54.1 \%$ of SN-38 lactone in the supernatant was bound to protein. At $1 \mathrm{~h}$ after the start of incubation, $80.4 \%$ of SN-38 in the supernatant (SN-38 lactone, $54.4 \%$ and $\mathrm{SN}-38$ carboxylate, $8.3 \%$ ) was bound to protein. At $3 \mathrm{~h}$ after the start of incubation, the bulk

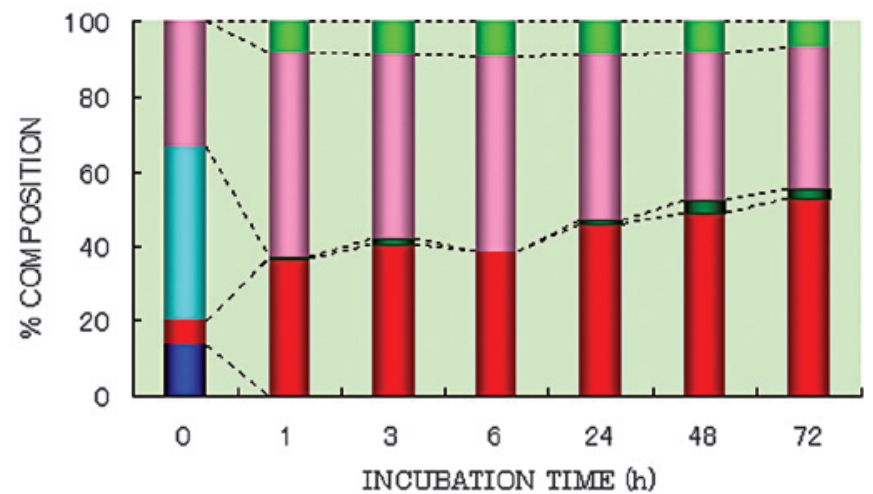

Figure 1. Change in the percentage composition of SN-38 glucuronide during the course of incubation in 10-fold diluted cecal content culture. SN-38 glucuronide was added to the culture medium and incubated anaerobically with $\mathrm{N}_{2}$ for up to 72 h. Dark blue, SN-38 glucuronide in pelleted fraction; light blue, SN-38 glucuronide in supernatant; red, SN-38 lactone in pelleted fraction; pink, SN-38 lactone in supernatant; dark green, SN-38 carboxylate in pelleted fraction; light green, SN-38 carboxylate in supernatant.

of the $\mathrm{SN}-38$ was bound to protein, and the percentage of the protein-bound form was relatively constant (85.8 to $91.7 \%$ ). The congregated lactone gradually moved from the supernatant to the pelleted fraction, with the percentage of the lactone form in the supernatant gradually decreasing from $54.4 \%$ after $1 \mathrm{~h}$ to $37.8 \%$ after $72 \mathrm{~h}$ of incubation. This decrease may be attributable to a transition from the aqueous solution to the pelleted fraction due to precipitation of the protein-bound lactone.

\section{Discussion}

We have previously shown that little SN-38 was found in the supernatant when SN-38 lactone was added to growth medium, and that a significant quantity was rapidly adsorbed by intestinal bacterial cell walls in the pelleted fraction (28). In this experiment, extensive deconjugation of $\mathrm{SN}-38 \mathrm{G}$ to 
SN-38 occurred in mixed cecal cultures carried out under anaerobic growth conditions, as reported by Takasuna et al (22). As a result, almost all of the SN-38 metabolites found in the test tubes were deconjugated after $1 \mathrm{~h}$ of incubation (Fig. 1). The cytotoxic mechanism of SN-38 was originally ascribed to the closed lactone form, and opening of the lactone ring (carboxylate form) results in the loss of anticancer activity (29). In the present study, at $1 \mathrm{~h}$ after the start of incubation, $91.4 \%$ was found in the active lactone form of SN-38 $(37.0 \%$ in the pelleted fraction and $54.4 \%$ in the supernatant) and $8.6 \%$ in the carboxylate form; in other words, $62.7 \%$ of the added SN-38G was found in the supernatant (Table I). Furthermore, $80.4 \%$ of the $\mathrm{SN}-38$ in the supernatant was bound to protein, and $19.6 \%$ was detected as the active free form of SN-38. In total, only $12.3 \%(19.6 \times 62.7 \%)$ of $\mathrm{SN}-38 \mathrm{G}$ added to the test tube was found in the supernatant as the ultrafiltrable, free non-protein-bound form. The percentage of free SN-38 gradually decreased from $12.3 \%$ after $1 \mathrm{~h}$ to $6.4 \%$ after $72 \mathrm{~h}$ of incubation. This decrease indicates that more than $90 \%$ of the SN-38G added to the growth medium was either adsorbed onto the bacterial cell membrane in the pelleted fraction or dietary fibers, or existed in the protein-bound form in the supernatant after $1 \mathrm{~h}$ of incubation. It is noteworthy that only approximately $10 \%$ of the SN-38G added to the growth medium existed in the free unbound form.

Deconjugation of $\mathrm{SN}-38 \mathrm{G}$ to $\mathrm{SN}-38$ by $\beta$-glucuronidase in the intestinal microflora is considered to be a major contributory factor in the intestinal toxicity of CPT-11, which is believed to result from contact of this deconjugated SN-38 with the intestinal mucosa $(11,19-24)$. Unlike other camptothecin drugs, the SN-38 lactone form preferentially binds to blood proteins as compared with the corresponding carboxylate form, resulting in significant stability in the presence of human plasma (30). Furthermore, since the lactone form of SN-38 is highly water-insoluble, SN-38 is rapidly and tightly bound to hydrophobic bacterial cell walls or dietary fibers (Fig. 1) (28), which may explain the reason that little free $\mathrm{SN}-38$ remained in the lactone form in the supernatant of the 10-fold diluted culture in this study. This event potentially reduces the availability of the active metabolite, $\mathrm{SN}-38$, to the intestinal mucosa.

Hidaka et al (12) studied the adsorption of CPT-11 onto oral adsorbent AST-120 (Kremezin) for the prevention of delayed-onset diarrhea. It was presumed that the camptothecin drug may not have been completely adsorbed onto the Kremezin, since it failed to completely prevent diarrhea. A preliminary study by Takasuna et al (31) did not confirm the anti-diarrheal activity of D-glutaric acid-1,4-lactone monohydrolate, a specific $\beta$-glucuronidase inhibitor. However, the dose of D-glutaric acid-1,4-lactone was sufficient to inhibit $\beta$-glucuronidase. CPT-11-induced delayed-onset diarrhea is presumed to be attributable to damage to the small intestine, rather than the cecum, where $\beta$-glucuronidase activity in the lumen is highest. In their study, Fittkau et al (32) reported that D-saccharic acid 1.4-lactone alleviated CPT-11-induced mucosal damage in the small intestine, the luminal contents of which almost completely lack $\beta$-glucuronidase (22). Therefore, these authors suggested that a mechanism other than $\beta$-glucuronidase inhibition in the intestinal lumen may be involved (31). Furthermore, Kurita et al (33) carried out a
CPT-11-induced diarrhea study using Gunn rats, which have an inherent deficiency of uridine diphosphate glucuronosyl transferase $1 \mathrm{~A}$ and cannot conjugate $\mathrm{SN}-38$ to SN-38G. The onset of CPT-11-induced diarrhea in Gunn rats was not affected by $\beta$-glucuronidase activity. The alleviation of diarrhea by streptomycin in the Gunn rats indicated that streptomycin exerted its effect by a mechanism other than the inhibition of $\beta$-glucuronidase.

In conclusion, the results of the present study have shown that the extensive conversion of $\mathrm{SN}-38 \mathrm{G}$ to $\mathrm{SN}-38$ occurred in mixed cecal content cultures grown under anaerobic growth conditions. Due to the highly hydrophobic nature of SN-38, a significant quantity of $\mathrm{SN}-38$ was adsorbed onto the intestinal microflora and dietary fibers in the pelleted fraction, or became bound to protein in the cell-free supernatant. Additionally, only $10 \%$ of SN-38 was detected as the protein-unbound form in the supernatant in vitro. Further studies are therefore required to identify the exact role of the intestinal microflora in CPT-11-induced late-onset diarrhea.

\section{References}

1. Hsiang YH, Hertzberg R, Hecht S and Liu LF: Camptothecin induces protein-linked DNA breaks via mammalian DNA topoisomerase I. J Biol Chem 260: 14873-14878, 1985.

2. Masuda N, Kudoh S and Fukuoka M: Irinotecan (CPT-11): pharmacology and clinical applications. Crit Rev Oncol Hematol 24: 3-26, 1996.

3. Hecht JR: Gastrointestinal toxicity or irinotecan. Oncology (Williston Park) 12: 72-78, 1998.

4. Pizzolato JF and Saltz LB: The camptothecins. Lancet 361: 2235-2242, 2003.

5. Abigerges D, Armand JP, Chabot GG, et al: Irinotecan (CPT-11) high-dose escalation using intensive high-dose loperamide to control diarrhea. J Natl Cancer Inst 86: 446-449, 1994.

6. Mori K, Kondo T, Kamiyama Y, Kano Y and Tominaga K: Preventive effect of Kampo medicine (Hangeshashin-to) against irinotecan-induced diarrhea in advanced non-small-cell lung cancer. Cancer Chemother Pharmacol 51: 403-406, 2003.

7. Kehrer DF, Sparreboom A, Verweij J, et al: Modulation of irinotecan-induced diarrhea by cotreatment with neomycin in cancer patients. Clin Cancer Res 7: 1136-1141, 2001.

8. Alimonti A, Satta F, Pavese I, Burattini E, Zoffoli V and Vecchione A: Prevention of irinotecan plus 5-fluorouracil/ leucovorin-induced diarrhoea by oral administration of neomycin plus bacitracin in first-line treatment of advanced colorectal cancer. Ann Oncol 14: 805-806, 2003.

9. Furman WL, Crews KR, Billups C, et al: Cefixime allows greater dose escalation of oral irinotecan: a phase I study in pediatric patients with refractory solid tumors. J Clin Oncol 24: 563-570, 2006

10. Takeda Y, Kobayashi K, Akiyama Y, et al: Prevention of irinotecan (CPT-11)-induced diarrhea by oral alkalization combined with control of defecation in cancer patients. Int $\mathrm{J}$ Cancer 92: 269-275, 2001.

11. Michael M, Brittain M, Nagai J, et al: Phase II study of activated charcoal to prevent irinotecan-induced diarrhea. J Clin Oncol 22: 4410-4417, 2004.

12. Hidaka M, Yamasaki K, Okumura M, et al: Adsorption of irinotecan onto oral adsorbent AST-120 (Kremezin) for preventing delayed diarrhea. Cancer Chemother Pharmacol 59: 321-328, 2007.

13. Ychou M, Douillard JY, Rougier P, et al: Randomized comparison of prophylactic antidiarrheal treatment versus no prophylactic antidiarrheal treatment in patients receiving CPT-11 (irinotecan) for advanced 5-FU-resistant colorectal cancer: an open-label multicenter phase II study. Am J Clin Oncol 23: 143-148, 2000.

14. Savarese DM, Halabi S, Hars V, et al: Phase II study of docetaxel, estramustine, and low-dose hydrocortisone in men with hormone-refractory prostate cancer: a final report of CALGB 9780. Cancer and Leukemia Group B. J Clin Oncol 19: 2509-2516, 2001 . 
15. Lenfers BH, Loeffler TM, Droege CM and Hausamen TU: Substantial activity of budesonide in patients with irinotecan (CPT-11) and 5-fluorouracil induced diarrhea and failure of loperamide treatment. Ann Oncol 10: 1251-1253, 1999.

16. Iyer L, King CD, Whitington PF, et al: Genetic predisposition to the metabolism of irinotecan (CPT-11). Role of uridine diphosphate glucuronosyltransferase isoform 1A1 in the glucuronidation of its active metabolite $(\mathrm{SN}-38)$ in human liver microsomes. J Clin Invest 101: 847-854, 1998.

17. Rivory LP and Robert J: Identification and kinetics of a beta-glucuronide metabolite of SN-38 in human plasma after administration of the camptothecin derivative irinotecan. Cancer Chemother Pharmacol 36: 176-179, 1995

18. Itoh T, Takemoto I, Itagaki S, Sasaki K, Hirano T and Iseki K: Biliary excretion of irinotecan and its metabolites. J Pharm Pharm Sci 7: 13-18, 2004

19. Araki $E$, Ishikawa $M$, Iigo $M$, Koide $T$, Itabashi $M$ and Hoshi A: Relationship between development of diarrhea and the concentration of SN-38, an active metabolite of CPT-11, in the intestine and the blood plasma of athymic mice following intraperitoneal administration of CPT-11. Jpn J Cancer Res 84 697-702, 1993

20. Ikegami T, Ha L, Arimori K, et al: Intestinal alkalization as a possible preventive mechanism in irinotecan (CPT-11)-induced diarrhea. Cancer Res 62: 179-187, 2002.

21. Ikuno N, Soda H, Watanabe M and Oka M: Irinotecan (CPT-11) and characteristic mucosal changes in the mouse ileum and cecum. J Natl Cancer Inst 87: 1876-1883, 1995.

22. Takasuna K, Hagiwara T, Hirohashi M, et al: Involvement of beta-glucuronidase in intestinal microflora in the intestinal toxicity of the antitumor camptothecin derivative irinotecan hydrochloride (CPT-11) in rats. Cancer Res 56: 3752-3757, 1996.

23. Alimonti A, Gelibter A, Pavese I, et al: New approaches to prevent intestinal toxicity of irinotecan-based regimens. Cancer Treat Rev 30: 555-562, 2004

24. Saliba F, Hagipantelli R, Misset JL, et al: Pathophysiology and therapy of irinotecan-induced delayed-onset diarrhea in patients with advanced colorectal cancer: a prospective assessment. J Clin Oncol 16: 2745-2751, 1998.
25. Holdeman LV, Cato EP and Moore WEC (eds): Anaerobe Laboratory Manual, 4th edition. Virginia Polytechnic Institute and State University, Blacksburg, 1977.

26. Kurita A and Kaneda N: High-performance liquid chromatographic method for the simultaneous determination of the camptothecin derivative irinotecan hydrochloride, CPT-11, and its metabolites SN-38 and SN-38 glucuronide in rat plasma with a fully automated on-line solid-phase extraction system, PROSPEKT. J Chromatogr B Biomed Sci Appl 724: 335-344, 1999.

27. Kaneda N, Kurita A, Hosokawa Y, Yokokura T and Awazu S: Intravenous administration of irinotecan elevates the blood beta-glucuronidase activity in rats. Cancer Res 57: 5305-5308, 1997.

28. Yamamoto M, Kurita A, Asahara $\mathrm{T}$, et al: Metabolism of irinotecan and its active metabolite $\mathrm{SN}-38$ by intestinal microflora in rats. Oncol Rep 20: 727-730, 2008.

29. Beretta GL and Zunino F: Relevance of extracellular and intracellular interactions of camptothecins as determinants of antitumor activity. Biochem Pharmacol 74: 1437-1444, 2007.

30. Burke TG and Mi Z: The structural basis of camptothecin interactions with human serum albumin: impact on drug stability. J Med Chem 37: 40-46, 1994.

31. Takasuna K, Hagiwara T, Watanabe K, et al: Optimal antidiarrhea treatment for antitumor agent irinotecan hydrochloride (CPT-11)-induced delayed diarrhea. Cancer Chemother Pharmacol 58: 494-503, 2006.

32. Fittkau M, Voigt W, Holzhausen HJ and Schmoll HJ: Saccharic acid 1.4-lactone protects against CPT-11-induced mucosa damage in rats. J Cancer Res Clin Oncol 130: 388-394, 2004.

33. Kurita A, Kado S, Matsumoto T, et al: Streptomycin alleviates irinotecan-induced delayed-onset diarrhea in rats by a mechanism other than inhibition of beta-glucuronidase activity in intestinal lumen. Cancer Chemother Pharmacol 67: 201-213, 2011. 